\title{
MRI Compatibility of Robot Actuation Techniques - A Comparative Study
}

\author{
Gregory S. Fischer ${ }^{1, \star}$, Axel Krieger ${ }^{1, \star}$, Iulian Iordachita ${ }^{1}$, Csaba Csoma ${ }^{1}$, \\ Louis L. Whitcomb ${ }^{1}$, and Gabor Fichtinger ${ }^{1,2}$ \\ ${ }^{1}$ Center for Computer Integrated Surgery, Johns Hopkins University, USA \\ $\{$ gfischer, axel, iordachita, csoma, $11 w\} @ j h u . e d u$ \\ ${ }^{2}$ School of Computing, Queens University, Kingston, ON, Canada \\ gabor@cs.queensu.ca
}

\begin{abstract}
This paper reports an experimental evaluation of the following three different MRI-compatible actuators: a Shinsei ultrasonic motor, a Nanomotion ultrasonic motor and a pneumatic cylinder actuator. We report the results of a study comparing the effect of these actuators on the signal to noise ratio (SNR) of MRI images under a variety of experimental conditions. Evaluation was performed with the controller inside and outside the scanner room and with both $1.5 \mathrm{~T}$ and 3T MRI scanners. Pneumatic cylinders function with no loss of SNR with controller both inside and outside of the scanner room. The Nanomotion motor performs with moderate loss of SNR when moving during imaging. The Shinsei is unsuitable for motion during imaging. All may be used when motion is appropriately interleaved with imaging cycles.
\end{abstract}

\section{Introduction}

This paper reports an experimental evaluation of the following three different MRI-compatible actuators: a Shinsei ultrasonic motor, a Nanomotion ultrasonic motor and a pneumatic cylinder actuator. The requirements for MRcompatibility include: 1) MR safety, 2) preservation of image quality, and 3) ability to operate unaffected by the scanner's electric and magnetic fields [1. Ferromagnetic materials must be avoided entirely because they cause image artifacts and distortion due to field inhomogeneities, and can pose a dangerous projectile risk. Non-ferromagnetic metals such as aluminum, brass, titanium, high strength plastic, and composite materials are permissible. The use of any conductive materials in the vicinity of the scanner's isocenter must be limited because of the potential for induced eddy currents to disrupt the magnetic field homogeneity.

A review of MRI compatible systems for image-guided interventions can be found in 2]. Robotic assistance has been investigated for guiding instrument placement in MRI beginning with neurosurgery [3] and later percutaneous interventions [4,5]. Chinzei et al. developed a general-purpose robotic assistant

\footnotetext{
^ First authorship shared between Axel Krieger and Gregory Fischer.
} 
for open MRI based upon Shinsei ultrasonic motors [1] that was subsequently adapted for transperineal intra-prostatic needle placement [6]. Krieger et al. reported a 2-DOF manually actuated mechanical linkage to aim a needle guide for transrectal prostate biopsy with MRI guidance [7]. Fischer et al. reported a compact pneumatically operated robot for automated transperineal prostate biopsy and brachytherapy [8]. Other recent developments in MRI-compatible mechanisms include pneumatic stepping motors on a needle puncture robot [9], the Innomotion commercial pneumatic robot for percutaneous interventions (Innomedic, Herxheim, Germany) and combined ultrasound and MR imaging [10].

An investigation into MR-compatibility of actuation techniques for functional imaging is reported in [1]. Developments in MR compatible motor technologies include Stoianovici et al. who describe a MR-compatible pneumatic stepper motor 12 . Suzuki et al. describes ultrasonic motor drive techniques that enhance MR compatibility in [13]. The feasibility of using piezoceramic motors for robotic prostate biopsy is described in [14].

This work presents a quantitative comparison of the MR-compatibility of different actuation methods to determine their suitability for use in MRI. Three different actuators were investigated with different controller locations in two different scanners with $1.5 T$ and $3 T$ field strengths under four imaging sequences. To the authors' knowledge, this is the first reported quantitative side-by-side comparison of the compatibility of these actuation techniques for MR.
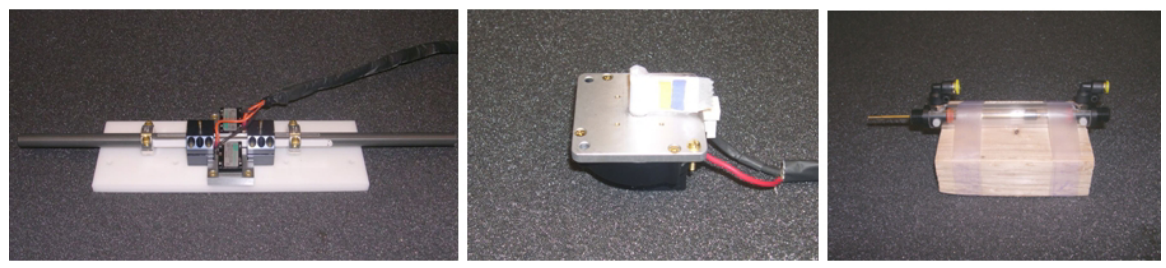

Fig. 1. The three actuators employed in this study: Nanomotion motor (left), Shinsei motor (center) and Pneumatic cylinder (right)

\section{Methods}

Experimental Setup: The motors selected for our experiments were: 1) Nanomotion linear piezoelectric non-magnetic motors HR2-1-N-10 (Nanomotion Ltd., Yokneam, Israel), 2) Shinsei rotary ultrasonic non-magnetic motor USR60-E3N (Shinsei Corporation, Tokyo, Japan), and 3) Customized Airpot pneumatic cylinders (Airpot, Norwalk, CT, USA).

The Nanomotion motor represents the class of linear piezoelectric motors including the PI PILine (Physik Instrumente, Karlsruhe, Germany) and Piezomotor Piezoleg (Piezomotor AB, Uppsala, Sweden). An opposing pair (maximum force $14 N$ ) was mounted to a linear guide consisting of a $16 \mathrm{~mm}$ diameter aluminum shaft supported with two DryLin R Adjustable Pillow Blocks (Igus GmbH, Koeln, Germany), as shown in Fig. 1 (left). During the experiments 

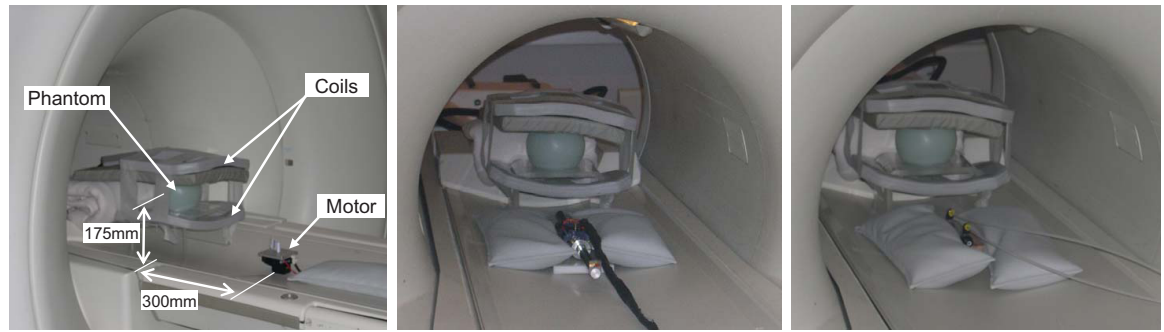

Fig. 2. General experimental setup for compatibility test in $1.5 \mathrm{~T}$ MR scanner with Shinsei motor (left), Nanomotion motor (center) and Pneumatic cylinder (right)

the motors were commanded to repeatedly translate for a displacement of $5 \mathrm{~cm}$ at a speed of $5 \mathrm{~cm} / \mathrm{s}$. The Shinsei motor is a high torque $(0.5 \mathrm{Nm})$, rotary ultrasonic motor that has been previously employed in MR-compatible systems [1]13. During the experiments, the motor was configured as shown in Fig. 1 (center). The motor was commanded to rotate at its minimum speed of $17 R P M$. The pneumatic cylinder employed in this study is specially designed for MR compatibility with a brass rod, graphite piston and glass cylinder as shown in Fig. 1 (right). The cylinders were repeatedly actuated with a pressure of 0.3bar and a speed of $8 \mathrm{~cm} / \mathrm{s}$. At least two speeds were initially evaluated for each motor, but speed was not found to significantly affect results, and was thus not included in this study.

The controller and its MR compatibility are described in 8. Inside an EMI shielded enclosure is an embedded computer, analog and digital I/O, pneumatic valves, and the respective amplifiers for the Nanomotion and Shinsei motors. External communication is via fiber optic Ethernet.

Compatibility was evaluated on a 3T Achieva scanner (Philips Medical Systems) and 1.5T Signa scanner (GE Healthcare). An $11 \mathrm{~cm}$ homogeneous spherical MR calibration phantom is placed in the isocenter on top of a plastic bridge $17.5 \mathrm{~cm}$ above the table surface. The motors are placed such that their center is at a horizontal distance of $30 \mathrm{~cm}$ from the center of the phantom. The general experimental setup is shown in Fig. 2. The distances were selected to avoid local susceptibility imaging artifact and mimic realistic actuator placement for most classes of robotic manipulators.

Two sets of experiments were performed in each of the two scanners for all three actuators. For the first set of experiments, the controller was placed inside the scan room, using the fiber optic communication link through a wave-guide from the control room. The only electrical connection to the controller is a filtered DC power supply through the penetration panel. For the second set of experiments the controller was placed inside of the control room and the motor cables and air hoses were passed through the wave-guide. Attempts failed to run both the Nanomotion motor and the Shinsei motor by connecting through D-sub connectors in the penetration panel, since the panel's integrated low-pass filters prevented the motors from functioning. Removing the built-in filter of the 
patch panel is not feasible in many clinical scanners and was not done for the reported experiments. For both motors, all cabling was enclosed in an additional shield with integrated ground strap. In the latter set, the strap was connected to ground at the penetration panel.

Each set of experiments consisted of the phantom being imaged alone (baseline) and consequently imaged in the presence of each actuator in power off configuration, power on configuration, and moving configuration.

Imaging Configuration: Four common MR sequences for diagnostic, real-time and functional imaging were selected to test the compatibility of the actuators (Table 1): T1 weighted fast gradient echo (T1 FGE/FFE) and T2 weighted fast spin echo (T2 FSE/TSE) sequences for diagnostic imaging, fast gradient echo (FSPGR/FGRE) sequence representing real-time imaging and spin echo-planar imaging (SE EPI) used for functional imaging.

Scanner gains, such as transmit gains, and analog and digital receiver gains, are normally set automatically during pre-scans before each image series. For a comparative study the gains need to be held constant for a particular imaging sequence throughout an experiment. The 1.5T GE scanner allowed for manual setting of the gains to the consistent values, thus ensuring comparability. The $3 \mathrm{~T}$ Philips scanner did not easily allow for manual gain setting; image series were therefore set up as a single dynamic series, with identical gains for all images within the same image sequence.

The receiving imaging coils used for the experiments were two channel medium size flex coil for the $3 \mathrm{~T}$ scanner and four channel cardiac coil for the $1.5 \mathrm{~T}$ scanner. Both coils consist of two panels. One panel was placed underneath the spherical phantom, the other one on top of the phantom (Fig. 2).

Compatibility Analysis: The MR-compatibility was evaluated based upon changes in signal to noise ratio (SNR). SNR is the signal in the center of the homogeneous phantom, divided by the noise intensity in the periphery as defined by the NEMA standard for determining SNR in MR images [15. The signal is defined as the mean pixel intensity in the region of interest (ROI). The noise is defined as the root mean square (RMS) signal intensity in an ROI outside of

Table 1. Scan Parameters for Compatibility Evaluation

\begin{tabular}{|c|c|c|c|c|c|c|c|c|}
\hline Protocol & Thickness & FOV & \# Slices & $\mathrm{TE}$ & $\mathrm{TR}$ & Flip Angle & NEX & Bandwidth \\
\hline \multicolumn{9}{|c|}{ 1.5T GE Signa } \\
\hline T1 FGE & $5 \mathrm{~mm}$ & $240 \mathrm{~mm}$ & 10 & $2.2 \mathrm{~ms}$ & $225 \mathrm{~ms}$ & $75^{\circ}$ & 1 & $977 \mathrm{~Hz} /$ pixel \\
\hline T2 FSE & $5 \mathrm{~mm}$ & $240 \mathrm{~mm}$ & 10 & $88 \mathrm{~ms}$ & $3000 \mathrm{~ms}$ & $90^{\circ}$ & 1 & $244 \mathrm{~Hz} /$ pixel \\
\hline FSPGR & $5 \mathrm{~mm}$ & $240 \mathrm{~mm}$ & 10 & $4.2 \mathrm{~ms}$ & $26 \mathrm{~ms}$ & $70^{\circ}$ & 1 & $244 \mathrm{~Hz} /$ pixel \\
\hline SE EPI & $5 \mathrm{~mm}$ & $240 \mathrm{~mm}$ & 10 & $45 \mathrm{~ms}$ & $187 \mathrm{~ms}$ & $90^{\circ}$ & 1 & $1953 \mathrm{~Hz} /$ pixel \\
\hline \multicolumn{9}{|c|}{ 3T Philips Achieva } \\
\hline T1 FFE & $5 \mathrm{~mm}$ & $240 \mathrm{~mm}$ & 3 & $2.3 \mathrm{~ms}$ & $225 \mathrm{~ms}$ & $75^{\circ}$ & 1 & $1075 \mathrm{~Hz} /$ pixel \\
\hline T2 TSE & $5 \mathrm{~mm}$ & $240 \mathrm{~mm}$ & 3 & $90 \mathrm{~ms}$ & $3000 \mathrm{~ms}$ & $90^{\circ}$ & 1 & $1036 \mathrm{~Hz} /$ pixel \\
\hline TFE/FGRE & $5 \mathrm{~mm}$ & $240 \mathrm{~mm}$ & 3 & $10 \mathrm{~ms}$ & $26 \mathrm{~ms}$ & $70^{\circ}$ & 1 & $1754 \mathrm{~Hz} /$ pixel \\
\hline SE EPI & $5 \mathrm{~mm}$ & $240 \mathrm{~mm}$ & 3 & $45 \mathrm{~ms}$ & $188 \mathrm{~ms}$ & $90^{\circ}$ & 1 & $656 \mathrm{~Hz} /$ pixel \\
\hline
\end{tabular}



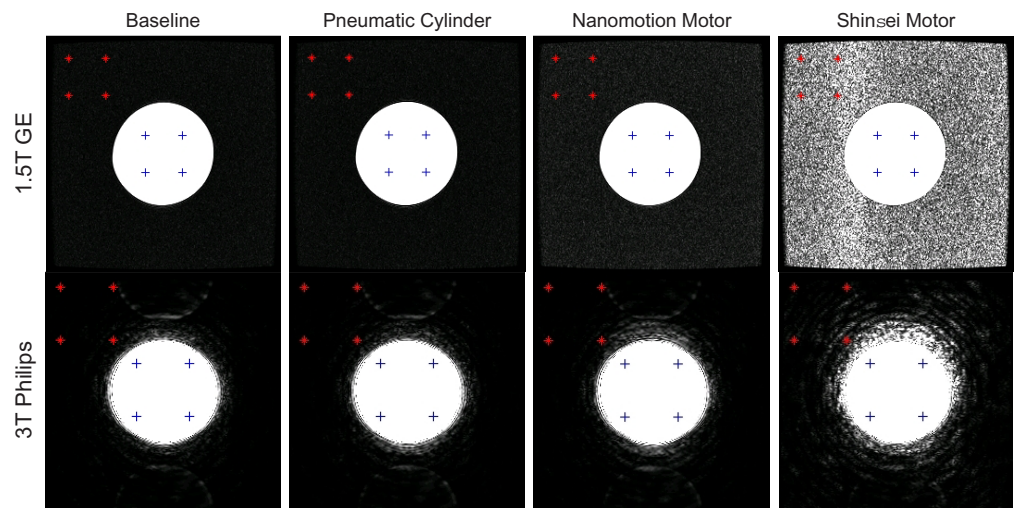

Fig. 3. Representative FGRE images for each motor type while moving under both 1.5T and 3T imaging. The ROI used in SNR calculations is represented by ' + ' and the noise ROI is represented by ' $*$ '. Window levels are identical across each row to compare motors, but different for each scanner - thus the perceived difference in image quality.

the phantom. Although a more standard method for defining noise is to use the standard deviation, the standard deviation does not accurately represent signal degradation in noise-saturated images such as that shown in Fig. 3 (top, right). SNR was evaluated at three slices for $3 \mathrm{~T}$ imaging and ten slices for $1.5 \mathrm{~T}$ imaging for each of the four imaging sequences under each configuration.

Representative FGRE images of the phantom, acquired with actuators moving are displayed in Fig. 3. The top row displays images of the $1.5 \mathrm{~T}$ scanner, the bottom row shows images obtained with the $3 \mathrm{~T}$ scanner. Window levels of the displayed images are identical for each row but differ for the two columns - thus the perceived difference in image quality between $1.5 \mathrm{~T}$ and $3 \mathrm{~T}$. The signal ROI used in SNR calculations is denoted by ' + ' and the noise ROI is denoted by '*'. The location of the noise ROI has a significant impact on calculated SNR; the selected ROI in the top-left corner minimizes variance between image slices and is outside of any ghosting artifacts present in the images.

\section{Results}

Fig. 4 shows the normalized SNR results for all sequences for the $1.5 \mathrm{~T}$ scanner, and Fig. 5 for the $3 \mathrm{~T}$ scanner. Each point in the graphs represents the mean SNR over all image slices, normalized to the mean baseline SNR. The top rows show normalized SNR for the three actuators with the controller inside the scan room, the bottom rows for the controller outside the scan room.

The following are the most significant observations:

- All four imaging sequences showed similar SNR behavior for both scanners.

- The SNR reduction due to the presence of actuators and controllers was greater on the $1.5 \mathrm{~T}$ scanner than on the $3 \mathrm{~T}$ scanner. 


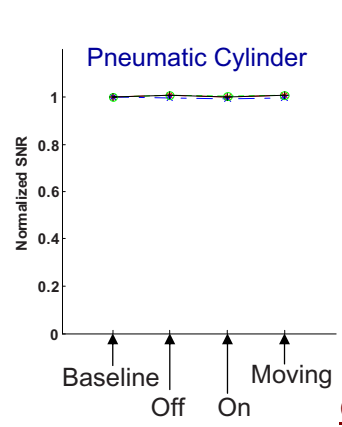

Controller Inside Scan Room
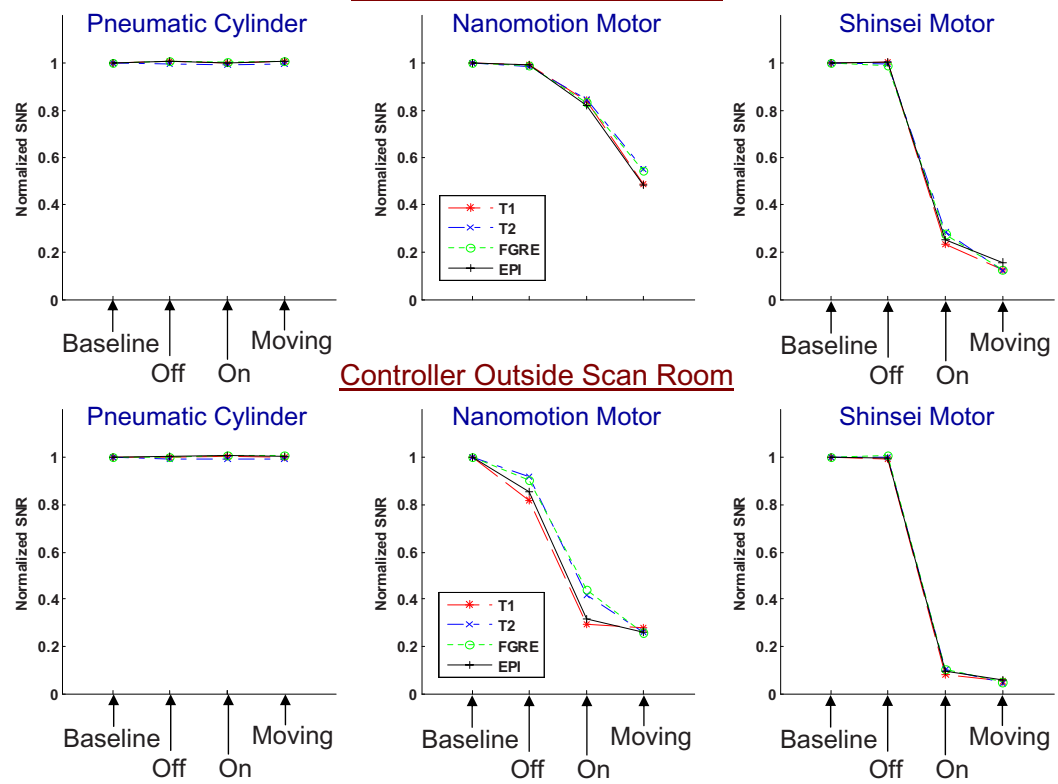

Controller Outside Scan Room
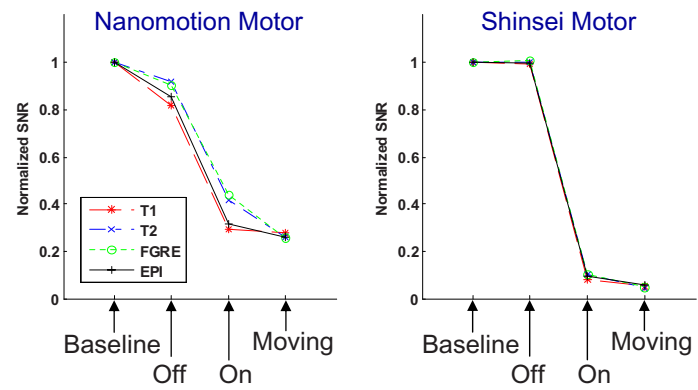

Fig. 4. SNR results under $\mathbf{1 . 5 T}$ imaging for each actuator in various configurations shown with the controller inside of the scanner room (top) and outside (bottom). SNR is normalized to one for the baseline in each set.

- Placing the controller inside the scanner room resulted in a reduction of SNR reduction compared to having the controller outside.

- The pneumatic cylinder and controller caused no negative impact on the SNR in all configurations.

- The Nanomotion motor reduced SNR moderately under 3T, especially with the controller placed in the scan room. Under 1.5T, reduction in SNR was significant. Actuator speeds did not significantly change the SNR.

- The Shinsei motor caused large reduction in SNR in all moving configurations. The noise increase nearly saturated the entire image with noise under 1.5T and caused significant noise ripples under 3T for the FGRE sequence (Fig. 3). Power on configuration reduced the SNR drastically.

Off-the-shelf D-sub capacitive and ferrite filters (DGFC9MF and DGF9MF respectively from L-Com, North Andover, MA) were evaluated on the control line for both the Nanomotion and Shinsei motors with the controller-inside configuration under $1.5 \mathrm{~T}$ imaging. The filters had minimal effect on Nanomotion. The capacitive filter provided significant SNR recovery for the Shinsei motor (from 90\% SNR reduction to $40 \%$ SNR reduction with respect to the baseline). 

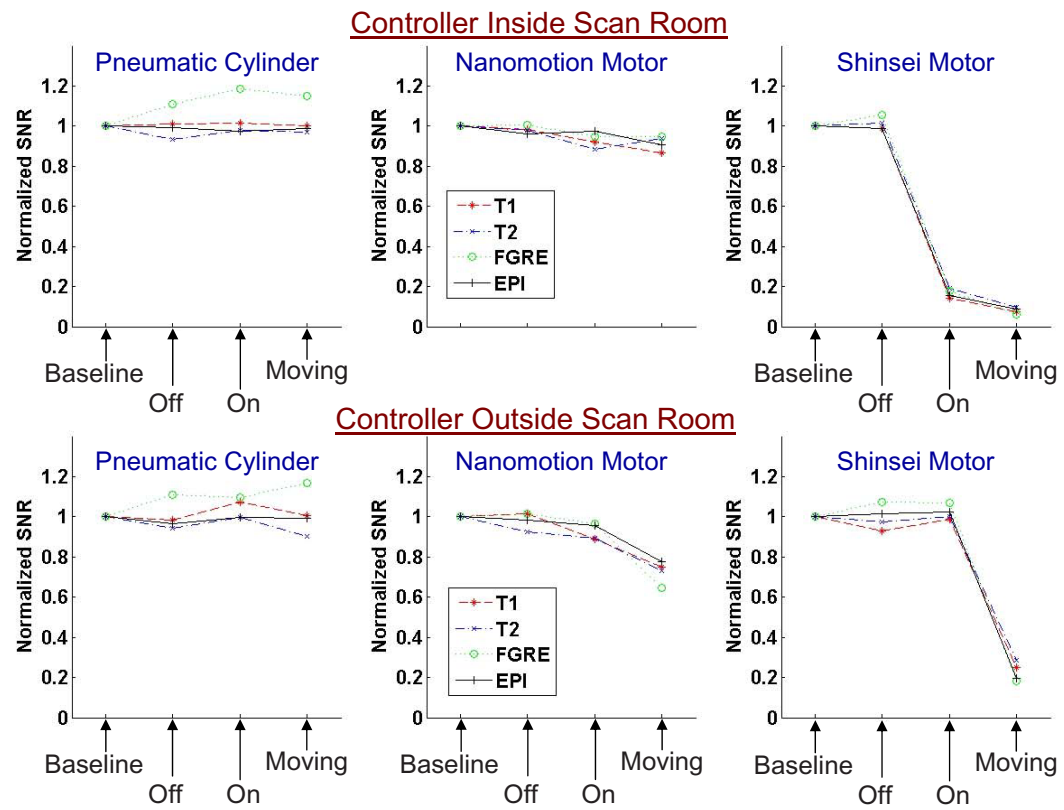

Fig. 5. SNR results under $\mathbf{3 T}$ imaging for each actuator in various configurations shown with the controller inside of the scanner room (top) and outside (bottom). SNR is normalized to one for the baseline in each set.

\section{Discussion}

The difference of SNR behavior for the two scanners cannot be attributed solely to the difference in field strength and frequency of the processing proton spins, used for imaging. The 1.5T scanner is an older model scanner, while the 3T scanner is newly installed. Improved shielding of the scan room, better integration of the wave-guides into the penetration panel, and reduction of the size of the fringe field were implemented on the newer scanner, thus potentially reducing the noise of the actuators independent of the field strength. Moreover, the different scanners had different imaging coil availability, which strongly impacts image quality. The differences in results between scanners demonstrate the necessity for testing mechatronic equipment in each scanner used.

Shinsei motors are less suitable for providing actuation during real-time imaging. If SNR is not a critical factor, the Nanomotion motors appear to be suitable for many applications; this is especially true if extra scan time (resulting in increased SNR) is a suitable compromise for image quality. For functional and other noise-critical imaging protocols, remote actuation such as pneumatics are preferred.

The results obtained here were not specifically optimized for one scanner or configuration. The authors felt that it was important to show the general applicability of each actuation method in a scanner-independent manner. Further, 
the filters were not optimized; it has been shown by Gassert, et al. [11] and Curiel, et al. [10] that it is possible to obtain significant SNR recovery with the use of specially designed filters. Similarly, shielding the motors may provide SNR recover, but this is not a generalizable nor commercially available option. In order to ensure scanner-independence, it is not possible to rely on the penetration panel due to variations in the built-in filtering and connector availability. Our experiences demonstrate the advantages for placing the controller inside the scanner room and communication through a fiber optic medium.

In summary: we reported the MR-compatibility of Shinsei, Nanomotion and pneumatic actuators under $1.5 \mathrm{~T}$ and $3 \mathrm{~T}$ imaging with controllers placed both inside and outside of the scanner room. All techniques demonstrate compatibility in the off configuration allowing the use of interleaved imaging and motion (e.g. 13]). The Shinsei motor did not allow for use during imaging; the Nanomotion performed with moderate SNR loss. Pneumatic actuation induced no significant degradation of image quality. SNR loss is reduced by placing an MR-compatible controller inside the scanner room. The study was designed to serve as a quantitative comparison of these actuation techniques under identical conditions. Although the results may not be generalized to all condition, they serve as an adequate baseline for comparing techniques.

Acknowledgements. We would like to thank Hugh Wall, Michael Schaer and Peter Guion for their help with the experiments. This work was supported by NSF EEC-97-31478, NIH RO1-CA111288-01, NIH RO1-EB002963 and CDMRP PCRP Fellowship W81XWH-07-1-0171.

\section{References}

1. Chinzei, K., Hata, N., Jolesz, F.A., Kikinis, R.: MR compatible surgical assist robot: system integration and preliminary feasibility study. In: Delp, S.L., DiGoia, A.M., Jaramaz, B. (eds.) MICCAI 2000. LNCS, vol. 1935, pp. 921-933. Springer, Heidelberg (2000)

2. Tsekos, N.V., Khanicheh, A., Christoforou, E., Mavroidis, C.: Magnetic resonancecompatible robotic and mechatronics systems for image-guided interventions and rehabilitation. In: An Rev BME, August 2007, vol. 9, pp. 351-387 (2007)

3. Masamune, K., Kobayashi, E., Masutani, Y., Suzuki, M., Dohi, T., Iseki, H., Takakura, K.: Development of an MRI-compatible needle insertion manipulator for stereotactic neurosurgery. J. Image Guid. Surg. 1(4), 242-248 (1995)

4. Felden, A., Vagner, J., Hinz, A., Fischer, H., Pfleiderer, S.O., Reichenbach, J.R., Kaiser, W.A.: ROBITOM-robot for biopsy and therapy of the mamma. Biomed. Tech (Berl) 47 (Suppl. 1 Pt. 1), 2-5 (2002)

5. Hempel, E., Fischer, H., Gumb, L., Höhn, T., Krause, H., Voges, U., Breitwieser, H., Gutmann, B., Durke, J., Bock, M., Melzer, A.: An MRI-compatible surgical robot for precise radiological interventions. In: CAS, April 2003, pp. 180-191 (2003)

6. DiMaio, S.P., Pieper, S., Chinzei, K., Fichtinger, G., Tempany, C., Kikinis, R.: Robot assisted percutaneous intervention in open-MRI. In: MRI Symp., p. 155 (2004) 
7. Krieger, A., Susil, R.C., Menard, C., Coleman, J.A., Fichtinger, G., Atalar, E., Whitcomb, L.L.: Design of a novel MRI compatible manipulator for image guided prostate interventions. In: IEEE TBME, February 2005, vol. 52, pp. 306-313 (2005)

8. Fischer, G.S., DiMaio, S.P., Iordachita, I., Fichtinger, G.: Development of a Robotic Assistant for Needle-Based Transperineal Prostate Interventions in MRI. In: MICCAI, November 2007, vol. 4791, pp. 425-433 (2007)

9. Taillant, E., Avila-Vilchis, J., Allegrini, C., Bricault, I., Cinquin, P.: CT and MR Compatible Light Puncture Robot: Architectural Design and First Experiments. In: Barillot, C., Haynor, D.R., Hellier, P. (eds.) MICCAI 2004. LNCS, vol. 3217, pp. 145-152. Springer, Heidelberg (2004)

10. Curiel, L., Chopra, R., Hynynen, K.: P2d-7 simultaneous us/mr imaging. In: Chopra, R. (ed.) Proc. IEEE Ultrasonics Symposium, pp. 1643-1646 (2006)

11. Gassert, R., Vanello, N., Chapuis, D., Hartwig, V., Scilingo, E., Bicchi, A., Landini, L., Burdet, E., Bleuler, H.: Active mechatronic interface for haptic perception studies with functional magnetic resonance imaging: compatibility and design criteria. In: ICRA 2006, pp. 3832-3837 (2006)

12. Stoianovici, D., Patriciu, A., Petrisor, D., Mazilu, D., Kavoussi, L.: A New Type of Motor: Pneumatic Step Motor. IEEE/ASME Trans. Mechatronics 12(1), 98-106 (2007)

13. Suzuki, T., Liao, H., Kobayashi, E., Sakuma, I.: Ultrasonic motor driving method for EMI-free image in MR image-guided surgical robotic system. In: IEEE IROS, pp. 522-527 (2007)

14. Elhawary, H., Zivanovic, A., Rea, M., Davies, B., Besant, C., McRobbie, D., de Souza, N., Young, I., Lampérth, M.: The feasibility of mr-image guided prostate biopsy using piezoceramic motors. In: Larsen, R., Nielsen, M., Sporring, J. (eds.) MICCAI 2006. LNCS, vol. 4190, pp. 519-526. Springer, Heidelberg (2006)

15. The Association of Electrical and Medical Imaging Equipment Manufacturers: Determination of Signal-to-Noise Ratio (SNR) in Diagnostic Magnetic Resonance Imaging, NEMA Standard Publication MS 1-2008 (2008) 